Yeeun Shim, ${ }^{1}$ Yoo-Na Kim, ${ }^{2}$ Seung-Tae Lee, ${ }^{3,{ }^{*}}$ Jung-Yun Lee, ${ }^{2}$ Sung Hoon Kim, ${ }^{2}$ Jong Rak Choi ${ }^{3}$

'Department of Laboratory Medicine, Graduate School of Medical Science, Brain Korea 21 Project, Yonsei University College of Medicine, Seoul, Korea

${ }^{2}$ Department of Obstetrics and Gynecology, Yonsei University College of Medicine, Seoul, Korea

${ }^{3}$ Department of Laboratory Medicine, Yonsei University College of Medicine, Seoul, Korea (lee.st@yuhs.ac)

Objective: Poly (ADP-ribose) polymerase (PARP) inhibitors are used to treat patients with defective homologous recombination repair (HRR), such as deleterious mutations in BRCA1 or BRCA2. Reversion mutation and the subsequent restoration of HRR pathway has been suggested as main resistance mechanism. However, reversion mutations do not fully explain the resistance mechanism. In this study, we enrolled PARP inhibitor treated ovarian cancer patients with germline or somatic BRCA mutation $(\mathrm{n}=71)$ and analyzed circulating tumor DNA (ctDNA) obtained from relapsed patients after progression on PARP inhibitor $(\mathrm{n}=16)$.

Methods: From whole blood samples, ctDNA was extracted from plasma and germline DNA from buffy coat. Extracted DNA was target enriched with panel targeting 531 cancerrelated genes, and the resultant library was sequenced using the Illumina Novaseq 6000 sequencing system. The sequencing data was analyzed using our custom analysis pipeline.

Results: Among 16 relapsed patients, 11 patients had both before PARP inhibitor treatment and relapsed samples, 5 patients had only relapsed sample. Every patient had tissue NGS data. Analysis of ctDNA samples showed ctDNA-specific variants. BRCA reversion was found in two patients (12.5\%). Analysis of patient-matched paired samples before and after progression on PARP inhibitor showed an increase in intratumoral heterogeneity in post-progression samples. New variants in genes that are linked to DNA repair pathway were found to be associated with increase tumor mutation burden. Conclusion: In this study, we demonstrated profiling of ctDNA, a less invasive assay, can efficiently offer information on tumor heterogeneity and predict PARP inhibitor resistance.

Poster (039)

Rare Tumors \& Metastatic Tumors

https://doi.org/10.3802/jgo.2021.32.S1.039

\section{Mature cystic teratoma of the ovary with carcinoid component: a case report}

Dolly Lubis, "Andi Darma Putra, Hartono Tjahjadi

Faculty of Medicine of Indonesian University, Dr. Cipto Mangunkusumo Hospital, Jakarta, Indonesia (doelhappy@gmail.com)
Objective: Learnig about clinical manifestation and diagnosis of mature teratoma cases with malignant transformation. Methods: A 36 years old woman, P2A0, was referred from a primary hospital because of post-operative pathological examination revealed a malignancy. Earlier, she underwent bilateral salpingo-oophorectomy (BSO) at a primary hospital for indication benign ovarian cyst. After BSO procedure, postoperative pathology result revealed: In both ovary, tumor tissue composed of ectoderm, mesoderm, and endoderm components with tumor cells nucleated round/oval, vesicular, relatively uniform, fine-grained chromatin resembling "salt and pepper, partially solid." Immunohistochemistry result training pattern was consistent with carcinoid tumors.

Results: In conclusion, histological according to mature ovarian teratoma with carcinoid.

Conclusion: Malignant transformation of benign ovarian tumors, including mature teratoma, is very rare. In addition, the clinical findings and pre-operative examinations did not help a lot to establish the diagnosis. Often the diagnosis was discovered unexpectedly in post-operative specimens such as happened in this case.

Poster (040)

Epithelial Ovarian Cancer including Borderline Tumor

https://doi.org/10.3802/jgo.2021.32.S1.040

\section{Single-arm, phase II study of niraparib and bevacizumab maintenance in platinum- sensitive, recurrent ovarian cancer previously treated with a PARP-inhibitor: KGOG3056/ NIRVANA-R}

\author{
Junsik Park, ${ }^{1}$ Myong Cheol Lim, ${ }^{2}$ Jae-Kwan Lee, ${ }^{3}$ Dae Hoon Jeong, \\ Se Ik Kim, ${ }^{5}$ Min Chul Choi, ${ }^{6}$ Byoung Gie Kim, ${ }^{7}$ Jung-Yun Lee, ${ }^{1, *}$ \\ 'Yonsei University College of Medicine, Seoul, Korea (jungyunlee@yuhs.ac) \\ ${ }^{2}$ National Cancer Center, Goyang, Korea \\ ${ }^{3}$ Korea University, Seoul, Korea \\ ${ }^{4}$ Inje University, Busan, Korea \\ ${ }^{5}$ Seoul National University College of Medicine, Seoul, Korea \\ ${ }^{6} \mathrm{CHA}$ Bundang Medical Center, Seongnam, Korea \\ ${ }^{7}$ Sungkyunkwan University School of Medicine, Suwon, Korea
}

Background: Given the expanding clinical use of poly(ADPribose) polymerase inhibitors (PARPis), there is a significant need for optimal strategies with which to treat patients whose cancer progresses while using a PARPi. However, the treatment consensus after PARPi has not been established. PARPi and anti-angiogenic agent combination therapy is a promising option to treat relapsed patients exposed to a PARPi. The synergistic efficacy of dual maintenance therapy 